\title{
LA CONSTRUCCIÓN DE LA IDENTIDAD PÚBLICA DEL MOVIMIENTO ANTIGLOBALIZACIÓN EN ESPAÑA
}

\author{
MANUEL JIMÉNEZ SÁNCHEZ \\ JAVIER ALCALDE VILLACAMPA* \\ IESA-CSIC. Córdoba \\ Instituto Juan March. Madrid \\ PALABRAS CLAVE ADICIONALES \\ Movimientos sociales, Protesta social, Medios \\ de comunicación, Globalización, Análisis del \\ discurso político. \\ ADDITIONAL KEYWORDS \\ Social Movements, Social Protest, Mass Media, \\ Globalization, Political Discourse Analysis.
}

RESUMEN. Desde Seattle, la atención prestada por los medios de comunicación al movimiento antiglobalización ha estado vinculada a la cuestión de la violencia. Su mayor presencia mediática ha impulsado su consolidación como movimiento global. También ha elevado el coste político de la represión fisica de las acciones no-violentas y ha fomentado un cambio gradual en la respuesta de las autoridades. En este sentido, el espacio central de confrontación parece haberse desplazado (o extendido) desde las calles al terreno de lo simbólico, para localizarse en el proceso de configuración de la imagen (o identidad) pública del movimiento a través del discurso en los medios. Este trabajo indaga en la naturaleza de este proceso mediante el análisis de los discursos recogidos en las noticias publicadas en El Pais durante la presidencia española de la UE en el año 2002.

SUMMARY. Since Seattle, the media attention to the antiglobalisation movement has been bound to the issue of public (dis) order. This greater media attention has promoted the consolidation of the movement. It has also increased the political costs of the physical repression of non-violent action and has led to the gradual modification of the authorities responses. In this sense, the locus of contention has moved (or expanded) from the streets to the symbolic domain, to a focus on the process of configuration of its public identity (or image) through media discourse. This paper inquires into the nature of this process through the analysis of discourses displayed in the news published in $E l$ Pais during the Spanish Presidency of the EU in 2002.

*Agradecemos a Victor Sampedro que nos animara a escribir este trabajo así como la revisión crítica que ha realizado del texto.

E-mail:mjimenez@iesaa.csic.es jalcalde@ceacs.march.es

Revista Internacional de Sociología (RIS)

Tercera Época, $n^{\circ} 33$, Septiembre-Diciembre, 2002, pp. 211-235. 
RIS

REVISTA INTERNACIONAL DE SOCIOLOCIA

\section{INTRODUCCIÓN}

Desde su popularización tras Seattle, la visibilidad en los medios de comunicación del movimiento antiglobalización (MAG), ha estado vinculada a la violencia. La perspectiva de eventuales incidentes de orden público, alentada tanto por el desarrollo de cumbres internacionales anteriores, como por la creciente envergadura de los dispositivos de seguridad', ha aumentado la atención de los medios por unas cumbres que previamente recibían una cobertura más discreta. Con relación al MAG, esta circunstancia ha favorecido la toma de conciencia de colectividad (de compartir una identidad) de los diferentes grupos y, por tanto, ha impulsado su consolidación como movimiento global ${ }^{2}$. Al mismo tiempo, su presencia en los medios ha elevado también el coste de la represión física de sus acciones de protesta no-violenta. La experiencia de protestas posteriores a Seattle (Praga, Quebec, Barcelona, Génova, etcétera) ha producido un cambio gradual en la respuesta de las autoridades, así como en las estrategias del propio movimiento ${ }^{3}$. En este sentido, el espacio central de confrontación parece haberse desplazado de las calles al terreno de lo simbólico, para localizarse en el proceso de configuración de la imagen (o identidad) pública del movimiento a través del discurso en los medios. En esta coyuntura, no parece exagerado afirmar que el futuro del MAG se va a decidir, en primera instancia, en la batalla mediática sobre su identidad pública.

Este trabajo examina la naturaleza de los discursos mediáticos sobre el MAG, así como el tratamiento informativo de sus actividades y demandas. Para ello se analiza el discurso de las noticias sobre la presidencia española de la Unión Europea publicadas en El País durante el primer semestre de 2002.

La elección del periódico El País como fuente de la muestra obedece a criterios de calidad, amplitud de la cobertura territorial y difusión del medio ${ }^{4}$. La utilización

\footnotetext{
'Estas medidas de seguridad se han exacerbado tras el 11-S. En este sentido, y en relación con el Plan de Seguridad diseñado por el Ministerio del Interior durante la presidencia, el Director General de la Policía señalaba que "dos son los principales problemas a los que se enfrentará la policía: un posible atentado terrorista y la violencia de los grupos antisistema que acuden a las cumbres internacionales". Para hacer frente se contará con "el presupuesto que haga falta" (entrevista reproducida en www.policia.es/policiahoy).

${ }^{2}$ Una explicación de este salto cualitativo (y cuantitativo) en el MAG a partir de Seattle puede encontrarse en Smith, 2001.

${ }^{3}$ Desde finales de la década de los ochenta, las reuniones del Banco Mundial, el FMI y otros organismos internacionales venían siendo objeto de protestas, en ocasiones, acompañadas de represión policial e incidentes violentos (véanse, por ejemplo, Gerhards y Rucht 1992, Ericson y Doyle 1999). Sin embargo, es a partir de la reunión de Seattle de la OMC, en noviembre de 1999, cuando se inicia la fase mediática del MAG (Smith, 2001).

${ }^{4}$ El País puede ser considerado un periódico de calidad, adjetivo que permite distinguirlo de otros periódicos de información general de corte sensacionalista; con una línea editorial estable,
} 
de una sola fuente parcela nuestra aproximación al proceso de construcción de la identidad pública del MAG a los discursos que quedan registrados en las páginas de El País, con los sesgos que ello implica en cuanto a selección de noticias, fuentes, interlocutores, etc. Se trata pues de una aproximación parcial y sesgada, que sin restar valor alguno al ejercicio analítico propuesto, impone ciertas cautelas a la interpretación de los resultados.

\section{MOVILIZACIÓN SOCIAL, IDENTIDAD PÚBLICA Y MEDIOS DE COMUNICACIÓN}

Los medios de comunicación de masas desempeñan un papel clave en los procesos de acción colectiva. La dependencia de los medios ha significado una de las principales transformaciones en el contexto de los movimientos sociales (MS), en el último tercio del siglo XX, y ha incidido de manera específica en la naturaleza de su interacción con las autoridades (Gamson, 1990; Tarrow, 1998). Por un lado, prácticamente todos los aspectos de la movilización, desde la incorporación de activistas/socios, los formatos organizativos y las tácticas de protesta, se han visto crecientemente afectados por la presencia (real o potencial) de los medios ${ }^{5}$. Por otro lado, la mediatización de la acción colectiva ha afectado a la forma de la respuesta de las autoridades, alterando, entre otras cuestiones, la eficacia de la represión encubierta frente a la abierta (el recurso a la fuerza física). En líneas generales, se ha producido un cambio radical en las contiendas protagonizadas por MS, aumentando el papel del público y la intervención de terceros (aliados, mediadores, oponentes, etc.) (Gamson y Wolsfield, 1993; Moltoch, 1977).

Este proceso reclama de los investigadores mayor atención, aún si cabe, a la faceta simbólica de la confrontación social. En gran medida, la suerte de los MS se decide en el proceso de construcción de las definiciones de la realidad, en la que los medios constituyen una arena central. En este contexto, los discursos de los distintos actores están orientados a la definición de la identidad pública de los propios movimientos. El concepto de identidad pública, frente al carácter interactivo central en la idea de identidad colectiva, atiende al proceso mediante el cual, los MS persiguen imponer una imagen de sí mismos en lucha con la que intentan

ideológicamente cercana al PSOE, pero sin constituir un periódico de partido. Se trata del periódico de información general de mayor tirada e indice de lectura de España (véanse, por ejemplo, los resultados del Estudio General de Medios correspondientes al año 2002, http://www.aimc.es/aimc.php).

${ }^{5}$ Ya en 1970 Gusfield señala cómo la dependencia de la acción colectiva de interpretaciones compartidas de los hechos hace de las condiciones de comunicación una cuestión crucial para la vida de los MS (citado en Kielbowicz y Scherer 1986); véanse también Tarrow 1998, Grant y Maloney 1997). 
atribuirle otros actores incluidos los medios ${ }^{6}$. Como resultado de este proceso, la imagen pública dominante del movimiento determinará su legitimidad social y capacidad de movilización (Minkoff, 1997). En definitiva, la idea de identidad pública captura la imagen que el público ajeno al movimiento tiene de éste (y sobre la que basará sus decisiones de adhesión, rechazo, etc.), pero también atiende a la propia visión interna del movimiento (y sus aliados potenciales) y las pautas de interacción interorganizativas.

Desde esta perspectiva, el éxito de los MS depende de la medida en que son percibidos por el público como "cargados de razón" (a la hora de definir una situación como problema de interés general, identificar causas y proponer alternativas), como interlocutores "representativos" de los (amplios sectores de) afectados y sus métodos de protesta son considerados legítimos (lo que habitualmente implica la adopción de formas de protesta no violentas). A efectos analíticos podemos, pues, atender a tres dimensiones o elementos discursivos sobre los que es posible esperar que gire el proceso conflictual de configuración de la identidad pública de los MS en los medios: el fundamento de sus demandas, su representatividad y su naturaleza pacífica/violenta.

En este proceso, los medios no se limitan a describir los eventos acaecidos y transcribir el discurso emitido por sus protagonistas. Los medios deben ser analizados no sólo como arenas en la que se reproduce la confrontación de terceros, sino como un actor (crucial) más que, en función de sus intereses, genera su propio discurso al mismo tiempo que favorece a unos actores y discursos frente a otros (Page, 1996: 116) ${ }^{7}$. En definitiva, los medios son fundamentales en el proceso de acción colectiva; no sólo porque otorgan visibilidad a unas protestas frente a otras ${ }^{8}$, sino porque, además, pueden incidir sobre el propio proceso de movilización ${ }^{9}$ y son decisivos a la hora de definir la naturaleza (legítima) de la protesta y sus protagonistas ${ }^{10}$.

El análisis de la relación MS-medios se ha beneficiado ampliamente de las aportaciones desde la perspectiva del newsmaking, al incorporar consideraciones

\footnotetext{
${ }^{6}$ Para una distinción conceptual de las distintas nociones de identidad véase Johnston, Laraña y Gusfield 1994.

${ }^{7}$ Véase también Gamson y Wosfield 1993, Sampedro, 1998.

${ }^{8}$ Como señala Raschke, una protesta que no aparece en los medios no ha existido (citado en Rucht et al. 1998)

${ }^{9}$ Entre otras formas, por ejemplo, en su estudio sobre la mayor manifestación en la historia de Bélgica a raíz del caso Dutroux en 1996, Walgrave y Manssens (2000) señalan cómo, en ausencia de una base organizativa movilizadora, la prensa actuó como organización alternativa de movilización.

${ }^{10}$ En este sentido, en un trabajo clásico, Halloran et al. (1970) analizaron cómo una manifestación pacifica contra la guerra del Vietnam en Inglaterra fue transformada por los medios en un evento violento al centrarse en incidentes protagonizados por una minoría.
} 
relativas a la incidencia de las prácticas rutinarias de elaboración de la noticia y la tendencia ideológica de cada medio (van Zoonen 1992, Kielbowicz y Scherer 1986). En concreto, en el contexto de este trabajo, atendemos a dos aspectos de la lógica periodística que inciden en el sesgo de selección de las noticias. En primer lugar, la utilización predominante de fuentes oficiales o institucionalizadas y, por tanto, el acceso privilegiado del discurso establecido. Incluso cuando un MS es noticia, o entra en la agenda de los medios, la lógica que rige el trabajo periodístico privilegiará como portavoces a los sectores más institucionalizados del mismo o las elites políticas simpatizantes. Es decir, las demandas de un MS recibirán mayor atención cuanto más se articulen como objeto de confrontación política entre actores institucionalizados aunque esta situación no garantice su control sobre el discurso (sobre lo que de él o sus demandas se dice en los medios).

En segundo lugar, nuestro análisis es consciente de la primacía informátiva de lo excepcional, y en especial, el valor informativo de los eventos que implican violencia o problemas de orden público (Snyder y Killy, 1977; Hug y Wisler, 1998, Hocke, 1998; Jiménez, 2002). El uso de métodos disruptivos (o la amenaza de emplear los mismos) incrementa la probabilidad de recibir cobertura informativa. Sin embargo, esta circunstancia aumenta, al mismo tiempo, el riesgo de alimentar un discurso que imponga una imagen violenta del MS, lo que puede generar una dinámica de pérdida de apoyos $y$, por tanto, restar capacidad de incidencia.

\section{EL ANÁLISIS DE NOTICIAS DE PRENSA}

Las apreciaciones anteriores parten del presupuesto, bastante extendido en la ciencia política y la sociología, de que las realidades políticas se construyen con el discurso y por medio de él (Chilton y Schäffner, 2000). Entre las diversas funciones atribuibles al discurso, nuestro análisis se centra en la función estratégica de legitimación del mismo, que parte del supuesto de que la interpretación política (sobre el MAG) que se refleja en el discurso de los distintos actores (o emisores) puede ser considerada como parte coherente de una estrategia política más amplia hacia éste (Chilton y Schäffner, 2000)"

La unidad principal de análisis es la noticia de prensa. La noticia no se concibe como una mera traslación, más o menos fiel, de la realidad, sino como un marco a través del cual se construye rutinariamente el mundo social (van Dijk, 1996). 'En concreto, entendemos por noticias sobre la presidencia española de la Unión Europea (NPE) aquellas noticias que versan sobre la preparación, los contenidos y discusiones, y las decisiones alrededor del proceso político ligado a la presi-

\footnotetext{
${ }^{11} \mathrm{El}$ análisis del discurso que presentamos se basa fundamentalmente en un nivel semántico.
} 
R I S

REVISTA INTERNACIONAL DE SOCIOLOGIA

№ 33, Septiembre-Diciembre, 2002

MANUEL JIMÉNEZ Y JAVIER ALCALDE

dencia española y/o a las actividades del MAG en el marco de la presidencia española ${ }^{12}$.

La muestra comprende las NPE aparecidas en la edición diaria de la versión electrónica de El País entre el 16 de diciembre de 2001 y el 15 de julio de 2002, incluyendo las secciones de la edición estatal, así como las de Cataluña y Andalucía ${ }^{13}$. En total, la muestra ha sido extraida de la lectura de 210 ediciones diarias, en las cuales, de acuerdo con la definición de NPE, hemos identificado y codificado 610 noticias. De ellas, algo más de la mitad (328) aparecieron publicadas en las secciones de difusión estatal (Internacional, España, etcétera), mientras que en las secciones catalana y andaluza lo hacían un $15 \%$ (91) y un $32 \%$ (195), respectivamente ${ }^{14}$. Antes de proceder a presentar los resultados de nuestro análisis es conveniente introducir algunas notas sobre el MAG en España y, en concreto, sobre sus actividades durante la presidencia.

\section{EL MAG EN ESPAÑ ${ }^{15}$}

En España, al igual que ocurre en otros países, el MAG es el resultado de una amalgama de redes integradas por organizaciones de movimientos ecologistas, pacifistas, ONGS de desarrollo, sindicatos minoritarios, etcétera, así como entidades cuyo origen puede vincularse directamente a la lucha contra las políticas de organismos internacionales y a favor de una globalización alternativa. Entre estas últimas, una de las pioneras fue el Movimiento contra la Europa de Maastricht y la Globalización Económica (activo desde mediados de los noventa), al cual se han unido posteriormente otras como MRG-España, RCADE o ATTAC-España.

Antes del período analizado, el principal evento antiglobalización tuvo lugar en Barcelona, en junio de 2001, con motivo de la reunión anual del Banco Mundial,

\footnotetext{
12 Esta definición de noticia se centra en el discurso periodístico sobre los sucesos acontecidos, en el que quedan incluidos los artículos evaluativos (de fondo) y de opinión (incluidas las cartas al director). Sobre la noticia como discurso y su estudio véase van Dijk 1996.

${ }^{13}$ Como se indicó anteriormente, la elección de El País como fuente se fundamenta en los criterios de calidad, así como por su interés por la problemática de la globalización y el MAG. En su análisis hemos tenido en cuenta la tendencia ideológica y afinidades políticas del periódico.

${ }^{14} \mathrm{La}$ diferencia entre el volumen de NPE publicadas en las dos secciones autonómicas se explica en parte por la celebración posterior de la cumbre de Sevilla (y la mayor acumulación de noticias que sobre su celebración se producen). También deben ser consideradas cuestiones relacionadas con las peculiaridades de las dos redacciones y el distinto nivel de acceso de los representantes del MAG en cada caso.

${ }^{15}$ Sobre el MAG en España véanse Durán et al. 2001, Pastor, 2002, así como los sitios webs de las organizaciones del movimiento. Las direcciones de las aquí citadas son: www.nodo50.org/mast; www.mrg-e.org; www.rcade.org; www.attac.org/es.
} 
finalmente suspendida ante el miedo a que las movilizaciones hicieran fracasar el encuentro. No obstante, la organización de la contracumbre siguió adelante con los actos que tenían preparados. Durante la celebración de su acto central, la manifestación que congregó a 25.000 personas, se produjeron incidentes violentos provocados por una minoría de manifestantes y la polémica actuación policial.

En relación con el semestre de la presidencia europea de la UE, las actividades del MAG se organizaron en torno a las principales reuniones oficiales a celebrar, siendo las cumbres de jefes de Estado y de Gobierno de la UE de Barcelona (1516 de marzo) y Sevilla (21-22 junio) las citas que concentraron sus esfuerzos organizativos $^{16}$. En Barcelona el MAG estaba compuesto por tres plataformas diferenciadas. La Campaña contra la Europa del Capital y de la Guerra, principal promotor de las actividades durante la cumbre, como la multitudinaria manifestación a la que se sumaron las dos restantes plataformas: El Foro Social de Barcelona, que integra a la izquierda tradicional (incluido el PSC y los sindicatos) y la Plataforma Catalana. Esta última, escasamente vinculada a las anteriores, estaba integrada por organizaciones independentistas catalanas y Batasuna. La presencia del partido abertzale fue una de los principales argumentos para asociar el MAG con la violencia ${ }^{17}$.

En Sevilla (y en el resto de las citas del movimiento durante la campaña contra la presidencia española), es posible hablar de una correspondencia casi total entre MAG y Foro Social. Así, en Sevilla, el MAG estaba constituido básicamente por el Foro Social de Sevilla, con la participación de organizaciones de MS (ecologistas, estudiantiles, antimilitaristas, vecinales, etc.) y de grupos políticos de izquierda, como IU (en esta ocasión, ni el PSOE ni los sindicatos mayoritarios se integraron en el Foro).

El desarrollo de las principales convocatorias durante la presidencia se caracterizó por la masiva participación ciudadana y por la ausencia de incidentes violentos graves $^{18}$. Esta circunstancia, como mostraremos más adelante, ha favorecido la imagen pública del MAG.

\footnotetext{
${ }^{16}$ Sobre el MAG en España véanse Durán et al., 2001, Pastor 2002, así como los sitios webs de las organizaciones del movimiento. Las de las citadas aquí son: www.nodo50.org/mast; www.mrg-e.org; www.rcade.org; www.attac.org/es.

${ }^{17}$ Tanto que, en el conjunto de NPE, Batasuna aparece como la entidad más frecuentemente nombrada.

${ }^{18}$ En Barcelona se practicaron un total de 94 detenciones y cargas policiales sobre grupos radicales aislados. En la principal manifestación participaron 300.000 personas. En la de Sevilla la participación también fue extraordinaria (100.000 personas), no se registraron cargas policiales y sólo 3 detenciones. Otras 100.000 personas se manifestaron durante la cumbre de Madrid en mayo, la tercera reunión en importancia, sin que se produjesen incidentes.
} 
R I S

REVISTA INTERNACIONAL DE SOCIOLOGIA

№ 33, Septiembre-Diciembre, 2002

MANUEL JIMÉNEZ Y JAVIER ALCALDE

\section{LA EVOLUCIÓN DE LA COBERTURA DE LA PRESIDENCIA ESPAÑOLA DE LA UE}

En primer lugar, presentamos los resultados sobre la evolución de la cobertura informativa de la presidencia española y los temas que acapararon su atención. Nuestro interés se centra en valorar la presencia del MAG y sus demandas en El País.

El Gráfico 1 refleja la evolución del número de NPE en la muestra analizada. $\mathrm{El}$ mayor volumen de informaciones se produjo durante las principales reuniones de jefes de Estado y primeros ministros de la UE en Barcelona y Sevilla. Las noticias registradas en las secciones autonómicas se refieren casi exclusivamente a estos dos eventos, acentuando los dos picos informativos. El incremento (más suave en la línea del total) durante mayo refleja el desarrollo de la cumbre EUAmérica Latina-Caribe celebrada en Madrid (17-18 mayo).

Si la evolución en el volumen de la cobertura informativa nos señala los tres eventos políticos más relevantes que vertebraron la presidencia española ${ }^{19}$, podemos igualmente esperar que los temas tratados en las noticias indiquen las

Gráfico 1.

Evolución quincenal del número de NPE en El Pais

(16 diciembre 2001-15 julio 2002).

Total y desagregada por ediciones (estatal, Cataluña y Andalucia).

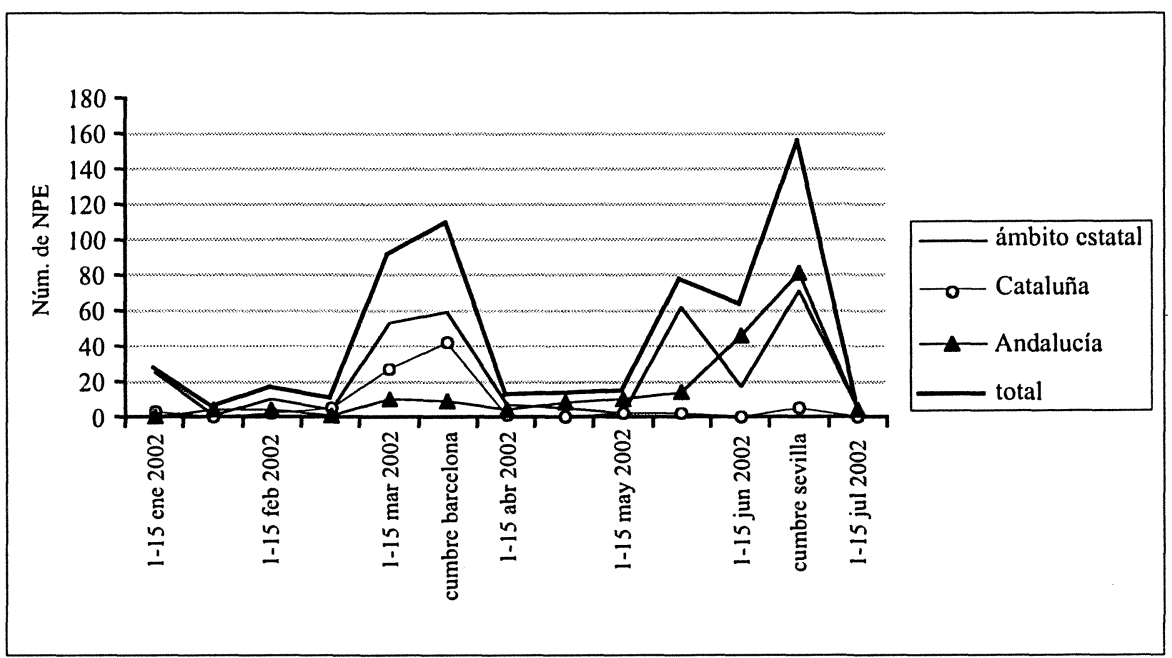


cuestiones más importantes en la agenda política y mediática. Así, al inicio de la presidencia las prioridades políticas se centraban en la lucha contra el terrorismo (seguridad), el impulso de la liberalización y modernización económica (principalmente del sector eléctrico), la introducción del euro, las negociaciones de la ampliación, el debate sobre el futuro de Europa y el fortalecimiento de la política exterior comunitaria ${ }^{20}$. En el transcurso del semestre, como consecuencia de los cambios en la coyuntura económica y política, esta agenda se modifica dando entrada a nuevos temas como la lucha contra la inmigración ilegal, el cual centraría gran parte del debate político en Sevilla ${ }^{21}$. En cuanto a la agenda mediática, junto a los temas sustantivos resulta razonable esperar un alto interés de la prensa por cuestiones relacionadas con los aspectos organizativos de las reuniones, así como por los asuntos domésticos que se entremezclan con los eventos de la presidencia ${ }^{22}$.

La tabla 1 muestra la frecuencia de los principales temas tratados en las NPE (columna 1). En cada noticia se codificaron hasta tres temas diferentes. Las distintas categorías temáticas han sido ordenadas en orden descendente según el porcentaje de NPE en las que son tratadas. Los valores en la columna 2 indican la extensión total, medida en palabras, de las NPE en las que cada uno de los temas aparece como el asunto principa ${ }^{23}$.

De acuerdo con las expectativas delineadas anteriormente, los temas relacionados con la organización de la presidencia son, efectivamente, los que con mayor frecuencia reciben tratamiento informativo y a los que un mayor número de palabras se ha dedicado en las páginas de El País. Del mismo modo, son frecuentes las noticias relativas a asuntos políticos domésticos. Estos resultados también reflejan las principales cuestiones en la agenda política del semestre español, especialmente aquéllas abordadas durante las reuniones de más alto nivel político. En este sentido, además del control de la inmigración ilegal, destacan las categorías de relaciones exteriores de la UE (con el conflicto de Oriente Próximo y Latinoamérica como principales cuestiones) o la preparación de la convención

\footnotetext{
${ }^{19}$ De acuerdo con informaciones policiales, 212 eventos directamente relacionados con la presidencia española tuvieron lugar durante este primer semestre de 2002 (Nieto, 2002).

${ }^{20}$ De acuerdo con la entrevista con J. Piqué (Ministro de Exteriores) del 2 de enero en El País.

${ }^{21}$ Mientras la liberalización fue uno de los principales temas tratados en Barcelona, en Sevilla, junto a la cuestión de la inmigración, se discutió acerca de la reforma de las instituciones, de la ampliación y sobre asuntos económicos, como el Pacto de Estabilidad (véase, por ejemplo, la entrevista con J. Piqué publicada el 19 de junio en El País).

${ }^{22}$ Como, por ejemplo, las relativas a la campaña electoral francesa, la reforma del paro, las relaciones Aznar-Zapatero, etc.

${ }^{23}$ Esta respuesta múltiple explica que la suma de porcentajes supere el $100 \%$. El criterio para seleccionar el tema principal y los restantes se fundamentó en las valoraciones de los investigadores sobre la extensión dedicada y su mención en los titulares.
} 
RIS

REVISTA INTERNACIONAL DE SOCIOLOCIA

№ 33, Septiembre-Diciembre, 2002

MANUEL JIMÉNEZ y JAVIER ALCALDE

Tabla 1.

La agenda informativa relativa a la presidencia española en El País. Temas por número de noticias y extensión.

\begin{tabular}{lcc}
\hline \multicolumn{1}{c}{ Temas } & $\begin{array}{c}\text { Porcentaje de NPE en } \\
\text { las que se trata el tema }\end{array}$ & $\begin{array}{c}\text { Extensión (suma } \\
\text { palabras de las NPE en } \\
\text { las que se trata el tema } \\
\text { como principal) }\end{array}$ \\
\hline Organización de la presidencia & 40,8 & 82178 \\
Seguridad/orden público & 37,0 & 77291 \\
Globalización alternativa & 17,2 & 31245 \\
Asuntos políticos domésticos & 12,0 & 21254 \\
Justicia y asuntos de interior (inmigración) & 11,3 & 23789 \\
Relaciones exteriores de la UE & 8,9 & 27046 \\
Reforma de la UE & 7,5 & 21007 \\
Liberalización (sector eléctrico) & 6,6 & 7497 \\
Economía y moneda (euro) & 4,6 & 6491 \\
Política exterior y de seguridad (defensa) & 4,6 & 8181 \\
Ampliación de la UE & 3,6 & 7656 \\
Resto & 16,5 & 25047 \\
Total temas & 170,6 & 338682 \\
Total noticias & $(1041)$ & $(610)$ \\
\hline
\end{tabular}

(reforma de la UE). Sin embargo, tanto en número de noticias como en extensión, el principal tema entre las cuestiones sustantivas de la agenda informativa, el control de la inmigración, resulta superado por dos cuestiones directamente vinculadas con el MAG: la seguridad durante la cumbre y el debate sobre los efectos negativos del proceso de globalización en curso (abordado, habitualmente, desde la perspectiva de la construcción europea).

Estos resultados sugieren que la presencia mediática del MAG, al menos en El País, también incluye sus demandas. El hecho de que casi dos de cada diez noticias versen sobre la crítica al modelo de globalización (y de construcción europea) es un claro indicador del alto grado de incidencia mediática del discurso crítico del MAG. Además, el amplio espacio que dedica El País a este tema en las secciones de ámbito estatal permite considerarlo como tema de interés general, confirmando el afianzamiento de la discusión sobre la globalización en la agenda mediática de este diario ${ }^{24}$.

\footnotetext{
${ }^{24}$ A pesar de que sólo el $50 \%$ de las NPE que tratan el tema de la globalización aparecen en las páginas de difusión estatal, en términos relativos se sitúa entre los temas que más espacio ocupan en las mismas.
} 
La existencia de un proceso de discusión y generación de opinión alrededor del proceso de globalización no sólo se refleja en la amplia cobertura mediática, sino que el tipo de NPE en las que se incluye esta información, ofrece igualmente un indicador importante para analizar el tratamiento mediático de la misma. La problemática de la globalización está presente en 34 artículos de opinión, la seguridad durante las cumbres en 46 , siendo los temas más recurrentes en este formato. Es decir, estas dos cuestiones han sido tratadas en aproximadamente un $30 \%$ y un $40 \%$, respectivamente, de los artículos de (creación de) opinión ${ }^{25}$.

La atención prestada a la seguridad durante la cumbre, no sólo constata el valor informativo de las cuestiones de orden público, sino que también señala el modo inicial de acceso del MAG a la opinión pública (i.e., la naturaleza del terreno sobre el que construye su imagen). Su consolidación en los medios es indiscutible: en un $84 \%$ del total de NPE encontramos referencias, más o menos directas, al $\mathrm{MAG}^{26}$. El fundamento de este acceso en la protesta (o la amenaza de la misma) es apreciable: en un $67 \%$ de las NPE se mencionan protestas protagonizadas por el MAG o se anuncian nuevas. Toda esta atención al MAG sugiere la existencia de un proceso en marcha de configuración de su imagen (de cuyo resultado puede depender, en gran parte, su futuro). En el siguiente apartado, el análisis de las NPE en las que se habla del MAG, nos permite analizar las dimensiones fundamentales en las que se centran los distintos discursos acerca de su identidad, así como la naturaleza y posicionamientos de los actores que participan en dicho proceso.

\section{LA CONFIGURACIÓN MEDIÁTICA DE LA IDENTIDAD PÚBLICA DEL MAG}

Las menciones al MAG o sus actividades de protesta no van siempre acompañadas de un discurso acerca de su naturaleza. No obstante, en un significativo $23 \%$ del total de noticias (i.e., $138 \mathrm{NPE}$ ) hemos identificado elementos de construcción de la imagen del MAG, ya sea en la reproducción que realiza El País del discurso de actores integrados en el MAG u otros más o menos afines al mismo, ya sea en el discurso del propio periódico (de sus redactores y columnistas). De ellas, 51 aparecen publicadas en la edición estatal, 33, en la catalana y 56, en la andaluza,

\footnotetext{
${ }^{25}$ Ambos temas son objeto de dos editoriales (de las 15 en total registradas como NPE).

${ }^{26}$ Ese porcentaje es el resultado de conjugar las noticias en las que encontramos una referencia directa $(40 \%)$ y aquéllas en las que se produce una referencia indirecta al MAG, a sus integrantes y/o a sus actividades de protesta (un $45 \%$ ). Si atendemos sólo a las noticias aparecidas en la sección de difusión estatal, el porcentaje de NPE en las que se realiza algún tipo de mención al MAG se mantiene en un significativo $48 \%$.
} 
R I S

REVISTA INTERNACIONAL DE SOCIOLOCIA

№ 33, Septiembre-Diciembre, 2002

MANUEL JIMÉNEZ Y JAVIER ALCALDE

lo que representa un $15 \%$, un $36 \%$ y un $29 \%$ respectivamente, del volumen total de NPE registradas en cada una de las tres ediciones.

\section{Evolución en el tiempo}

Como cabría esperar, las noticias con elementos discursivos sobre la identidad del MAG se concentran en los momentos de mayor intensidad informativa de marzo y junio, durante la celebración de las cumbres de Barcelona y Sevilla. Sin embargo, el peso relativo de este tipo de noticias es mayor aún en marzo, coincidiendo con la primera reunión de alto nivel, así como con la organización de la primera cumbre alternativa y la manifestación multitudinaria.

La evolución descrita por las columnas en el Gráfico 2 refleja esta variación en la intensidad del proceso de construcción de la identidad pública del'MAG en las páginas de El País. Las columnas apiladas distinguen las distintas ediciones donde fueron publicadas.

A pesar de que el número total de NPE es superior durante los últimos meses (véase Gráfico 1), el proceso de definición pública del MAG no sólo es más

Gráfico 2.

Evolución quincenal de NPE con elementos discursivos relativos a la naturaleza del $M A G$.

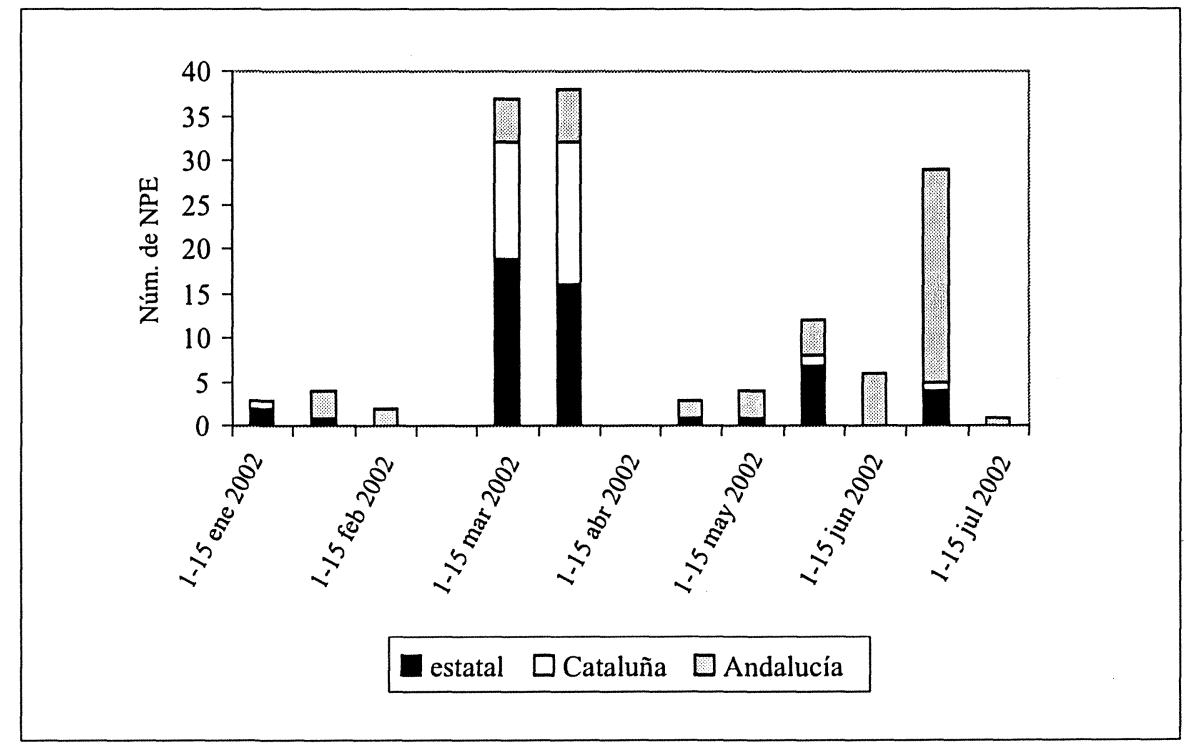


intensa durante marzo, sino que, además, se ubicó con mayor frecuencia en las secciones de difusión estatal. La menor intensidad de este proceso y, sobre todo, la pérdida de espacio en la edición estatal a partir de abril señalan a la cumbre de Barcelona como un momento crítico, de inflexión, en el proceso de configuración de su imagen pública.

Como hemos apuntado, en Barcelona se demostró la gran capacidad movilizadora del MAG. Junto con la manifestación de Génova, ha supuesto la mayor manifestación antiglobalización hasta el momento. Pero su éxito también puede evaluarse en la medida en que consiguió ofrecer una imagen pacífica. Por supuesto, dicha imagen fue potenciada o soslayada según qué medios. La prensa simpatizante (como El País) minimizó los incidentes violentos ${ }^{27}$. Los medios más conservadores y afines al Gobierno, por el contrario, centraron su (escasa) cobertura en la violencia ${ }^{28}$. Sin embargo, tras Barcelona los emisores a favor de una imagen positiva del MAG ganan argumentos en detrimento de sus opositores. Del mismo modo, la ausencia de Batasuna en la convocatoria de Sevilla, restó justificaciones al discurso de la violencia antiglobalizadora y allanó el camino a la visión contraria.

La evolución de otros indicadores refuerza la interpretación de estos resultados, que señalan la cumbre y contracumbre de Barcelona como el momento de mayor intensidad en la discusión sobre la identidad del MAG y de avance de su imagen no violenta. En este sentido, marzo registró tanto el mayor número de NPE con al menos una mención al MAG, como de emisores hablando de su naturaleza. Además, aunque la cumbre de Sevilla motivó de nuevo la multiplicación de las menciones al MAG, estas referencias estuvieron acompañadas con menor frecuencia de valoraciones. La violencia y otras dimensiones de la identidad pública del MAG sobre las que se centran los discursos mediáticos, así como la naturaleza de sus emisores, son objeto de análisis en las siguientes secciones.

\section{Las dimensiones tratadas sobre la identidad del movimiento}

Nuestra propuesta de estudio del proceso de construcción (conflictiva) de la identidad pública del MAG en la prensa se centra en el análisis del discurso de distintos actores (o emisores) con relación a tres dimensiones que consideramos críticas: su naturaleza violenta/pacífica, el grado de representatividad de sus integrantes y el fundamento de sus demandas. Las tres dimensiones resultaron estar presentes con relativa frecuencia en los pronunciamientos sobre el MAG recogidos en nuestra

\footnotetext{
${ }^{27}$ Desempeñando el papel inverso al descrito en Halloran et al., 1970; véase nota anterior.

${ }^{28}$ Sobre las distintas coberturas de la manifestación véase El País del 18 de marzo (edición de Barcelona).
} 
muestra. Así, un $55 \%$ de los emisores manifestó su opinión acerca de la naturaleza violenta/pacifica del MAG, un $42 \%$, de su representatividad, y un porcentaje similar $(41 \%)$ trató la cuestión del fundamento de sus críticas y/o alternativas. Estos porcentajes apenas cambian cuando se analizan sólo las noticias publicadas en la sección estatal; es decir, que cuando se habla de la naturaleza del MAG, los aspectos tratados son los mismos independientemente del nivel territorial de la edición. Dado el diferente nivel de acceso de los distintos tipos de emisores, este resultado sugiere también, en principio, que todos ellos han tendido a centrar su discurso en las mismas facetas de la identidad pública: en qué medida es un movimiento violento, representativo y fundamentado (aunque, como mostraremos más adelante, un análisis más detallado permite matizar esta afirmación de carácter general). El Gráfico 3 representa la evolución mensual de las frecuencias con la que los emisores tocaron cada una de las tres dimensiones.

Los resultados también sugieren que, proporcionalmente, las frecuencias de las tres dimensiones se mantienen estables en el tiempo. Es decir, la frecuencia con la que se habla de cada una respecto de las dos restantes es similar durante el semestre analizado. Tan sólo cabe destacar un ligero aumento de la tendencia a hablar del fundamento de las demandas del MAG (o su ausencia) después de la cumbre de Barcelona.

Gráfico 3.

Frecuencia con la que se valora la naturaleza violenta, la representatividad y el fundamento del MAG durante la presidencia española.

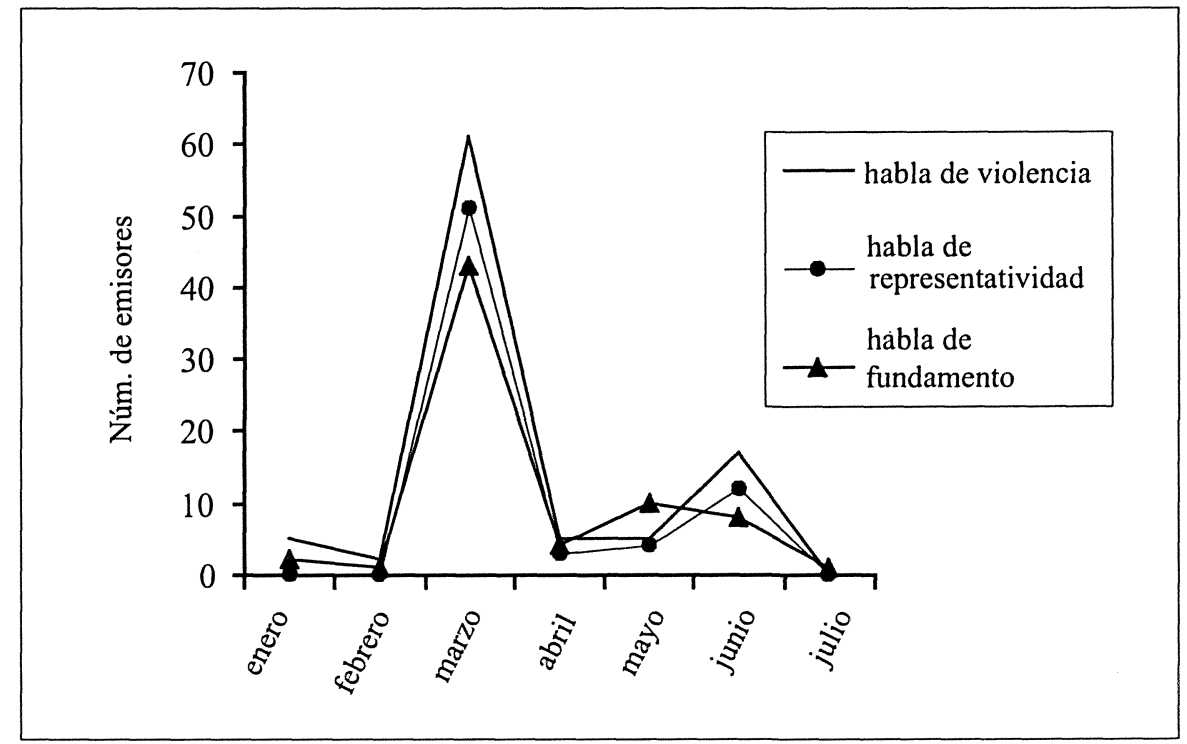




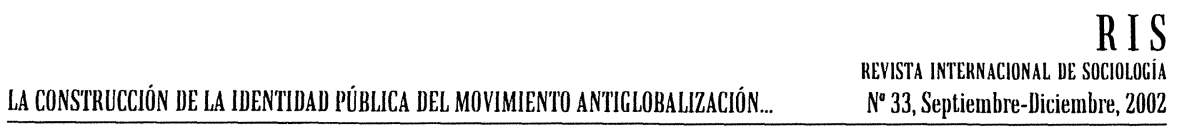

\section{Los actores en el proceso de construcción de la identidad pública del MAG}

Para analizar quién participa en el proceso de construcción de la imagen del MAG a través de las páginas de El País, en la tabla 2 hemos clasificado en siete categorías a los distintos tipos de emisores. Algunas de las categorías empleadas precisan de aclaración. Bajo la etiqueta de "El País" hemos incluido los casos en los que los propios periodistas o redactores de El País emiten juicios valorativos sobre el MAG (sin tratarse de transcripciones más o menos literales de declaraciones de otros actores). Las categorías Opinión 1 y Opinión 2 recogen los juicios realizados en artículos y columnas de opinión, agrupando en la primera a aquellos emisores colaboradores habituales, y en la segunda, a aquellos otros que no mantienen ningún vínculo con El País y que, en principio, pueden distanciarse de la línea editorial del periódico. Los resultados en las dos columnas reflejan la distribución porcentual de los emisores en todas las ediciones (columna 1) y sólo en la edición estatal (columna 2).

El distinto acceso de cada uno de los actores participantes en el proceso de construcción de la identidad pública del MAG refleja dos circunstancias tradicionalmente destacadas en los análisis del newsmaking. En primer lugar, la participación activa del periódico, a través de redactores y columnistas, en el proceso de configuración de la imagen del movimiento. En un $25 \%$ de estas noticias es el propio periodista quien actúa como emisor de enunciados acerca del MAG. Si a este porcentaje añadimos la actividad de columnistas y firmas habituales del

Tabla 2.

Naturaleza de los actores involucrados en la construcción de la identidad pública del MAG.

\begin{tabular}{lcc}
\hline Tipo de emisor & Todas las ediciones & Edición estatal \\
\hline El País (staff) & 20,8 & 30,2 \\
Opinión 1 (columnistas) & 16,1 & 19,0 \\
Opinión 2 & 8,9 & 3,2 \\
Políticos & 32,1 & 38,1 \\
MAG & 13,1 & 7,9 \\
Otros movimientos sociales & 3,6 & 1,6 \\
Otros & 5,4 & 0,0 \\
Total & $100,0(168)$ & $100,0(63)$ \\
\hline
\end{tabular}

Los porcentajes se refieren al total de emisores. Se han codificado hasta cuatro por NPE. 
RIS

REVISTA INTERNACIONAL DE SOCIOLOCIA

№ 33, Septiembre-Diciembre, 2002

MANUEL JIMÉNEZ Y JAVIER ALCALDE

periódico, comprobamos que prácticamente en la mitad de las noticias, al menos uno de los emisores registrados está ligado formalmente a El País y, por tanto, dentro de la línea editorial del mismo. El papel predominante del propio periódico se acentúa en la edición estatal donde los redactores y firmas habituales suponen la mitad de las emisiones de juicios de valor sobre el MAG.

En segundo lugar, los resultados también constatan el acceso privilegiado de los actores institucionalizados frente a los vinculados al sector de los movimientos sociales. Y, entre aquellos, las voces del PSOE frente a las del Gobierno y, especialmente, a las de otros partidos como IU. Los emisores dentro de la categoría de actores políticos institucionalizados constituyen un tercio del total. Estos resultados sugieren que el discurso mediático sobre el MAG ha entrado en el terreno de la confrontación política. La desigualdad en el acceso se amplía cuando excluimos del análisis las secciones autonómicas de El País. Sin embargo, cabe destacar la presencia de emisores del MAG (y, aunque más reducida, de otros MS) en las secciones de ámbito estatal.

\section{Aliados y opositores del MAG en la construcción de su identidad pública}

Las siguientes tablas presentan los resultados del análisis sobre los posicionamientos que los diferentes emisores mantuvieron con relación a las tres dimensiones sobre la identidad pública en las que se centra nuestro estudio. La clasificación de los emisores por categorías presenta dos variaciones significativas con respecto a la utilizada en la tabla 2. En primer lugar, las categorías El País y Opinión 1 han sido unificadas en una sola denominada El País. En segundo lugar, la categoría de actores políticos institucionalizados aparece desagregada de acuerdo a su adscripción partidista. Hay también una nueva categoría de emisores denominada "instancias mediadoras", que incluye actores ligados a la Administración de justicia y al Defensor del Pueblo. Las dos columnas a la derecha de las tablas ofrecen el número de emisores que tratan en su discurso la dimensión en cuestión y su porcentaje con relación al total de emisores dentro de cada categoría. Este porcentaje indica, pues, en qué medida cada tipo de emisor trata en su discurso (registrado por El País) las distintas dimensiones en las que se centra el proceso de construcción de la identidad pública del MAG. Las columnas a la izquierda presentan la distribución porcentual de los distintos emisores entre los distintos posicionamientos posibles.

Como puede observarse en la tabla 3, en el debate acerca del carácter violento de MAG ha participado un 55\% de los emisores. Se trata de la dimensión más frecuentemente tratada por los emisores registrados.

En términos absolutos, las categorías con mayor número de emisores son El País, seguida de Gobierno/PP y PSOE. La participación de emisores de El País es bastante similar en las distintas dimensiones de la identidad del MAG (como puede comprobarse en las siguientes tablas que presentaremos en esta 
Tabla 3.

La valoración de la naturaleza violenta del MAG en El Pais.

\begin{tabular}{|c|c|c|c|c|c|}
\hline \multirow[b]{2}{*}{ Emisores (\% de fila) } & \multicolumn{3}{|c|}{ El MAG ... } & \multirow[b]{2}{*}{$\begin{array}{l}\text { Núm. de } \\
\text { emisores }\end{array}$} & \multirow{2}{*}{$\begin{array}{c}\% \text { del total de } \\
\text { emisores en } \\
\text { cada } \\
\text { categoria }\end{array}$} \\
\hline & $\begin{array}{c}\text { es } \\
\text { violento }\end{array}$ & $\begin{array}{l}\text { engloba } \\
\text { minorias } \\
\text { violentas }\end{array}$ & $\begin{array}{c}\text { es } \\
\text { pacífico }\end{array}$ & & \\
\hline $\begin{array}{l}\text { El País } \\
\text { (staff, editorial y columnistas) }\end{array}$ & 3,4 & 55,2 & 41,4 & 29 & 46,8 \\
\hline $\begin{array}{l}\text { Opinión } \\
\text { (artículos y cartas al director) }\end{array}$ & 12,5 & 50,0 & 37,5 & 8 & 53,3 \\
\hline Gobierno/PP & 47,4 & 47,4 & 5,3 & 19 & 82,6 \\
\hline PSOE & - & 58,3 & 41,7 & 12 & 66,7 \\
\hline IU/LV & - & - & 100,0 & 1 & 33,3 \\
\hline $\mathrm{CiU}$ & 100,0 & & & 1 & 100,0 \\
\hline MAG & - & 12,5 & 87,5 & 8 & 36,4 \\
\hline Otros MS & - & - & 100,0 & 5 & 62,5 \\
\hline Instancias mediadoras & 66,7 & - & 33,3 & 3 & 60,0 \\
\hline Otros & 28,6 & 28,6 & 42,9 & 7 & 63,6 \\
\hline Total (o promedio) & 17,2 & 41,9 & 40,9 & 93 & 55,4 \\
\hline
\end{tabular}

sección $)^{29}$. No ocurre lo mismo en el caso del Gobierno/PP y del PSOE cuyo discurso se ha focalizado en la violencia. Como puede observarse en la columna de la derecha, respectivamente, un $82 \%$ y $66 \%$ de sus emisores abordaron la cuestión acerca de la violencia cuando se refirieron al MAG.

A partir de estas consideraciones, el resto de las columnas en la tabla 3 permiten dibujar un mapa bastante revelador del perfil de los aliados y opositores con los que ha contado el MAG durante el semestre de presidencia española de la UE. Las columnas centrales ofrecen la distribución porcentual de las frecuencias con las que los distintos emisores ( 93 en total) valoraron al MAG como esencialmente violento, pacífico, o en una posición intermedia. Precisamente, el discurso de un $42 \%$ apunta hacia esta imagen del MAG como un (amplio) movimiento que alberga en su seno a una minoría violenta. Un porcentaje similar lo define explícitamente como pacífico y, por último, el $17 \%$ restante lo considera globalmente violento.

\footnotetext{
${ }^{29}$ Es decir, el periódico es el actor que más habla de la naturaleza del MAG, sea cual sea la
} dimensión a la que atendamos. 
RIS

REVISTA INTERNACIONAL DE SOCIOLOGIA

Los emisores de El País no sólo son muy numerosos sino que, como podemos apreciar, han difundido una imagen pacífica del mismo (el $41 \%$ de las ocasiones) o que circunscribe la violencia a una minoría (el 55\% de las ocasiones). Estos resultados reflejan, además de la existencia de un interés mediático por el MAG, la adopción de una posición activa contraria al discurso que relaciona, sin matizaciones y en su conjunto, los antiglobalización con la violencia. Las opiniones reflejadas en la sección de opinión se mantienen también en esta línea. Aquí, el ligero aumento en la proporción de emisores que considera violento al movimiento puede indicar la naturaleza diferente de esta sección, potencialmente más abierta a posturas divergentes a la línea editorial del periódico. En suma, desde una perspectiva general, el discurso de El País con respecto a la naturaleza violenta o pacífica de los antiglobalización se mueve en la indefinición. El interés del periódico no se centra en esclarecer la naturaleza pacífica o violenta del MAG, ni en argumentar sobre la legitimidad o no de las estrategias de resistencia no-violenta o de desobediencia civil que configuran su repertorio de protesta. Un objetivo del periódico reside en atribuir al Gobierno conservador una actitud negativa hacia un movimiento que goza de creciente popularidad y aceptación social. De esta manera, el proceso de construcción de la identidad pública del propio MAG se ubica en el terreno de la confrontación partidista entre el Gobierno y el PSOE.

En este sentido, la cobertura que hace El País de las declaraciones de actores vinculados al Gobierno subraya un discurso que identifica claramente al MAG en su conjunto con la violencia y el desorden público (el único emisor registrado vinculado a $\mathrm{CiU}$ secunda esta visión). Contrarios a esta visión El País sitúa preferentemente a los emisores del PSOE. La frecuencia de los pronunciamientos desde las filas socialistas expresa su apoyo a las movilizaciones del MAG (en Barcelona el PSC estaba integrado en el Foro Social, y en Sevilla contaron con cierto apoyo desde el Gobierno andaluz) y su defensa ante las acusaciones conservadoras de estar apoyando a grupos violentos ${ }^{30}$. Junto al PSOE, aparecen emisores ligados al propio MAG y otros MS, y partidos de izquierdas (IU/LV). No obstante, como indican los porcentajes en la columna de la derecha, en comparación con otras dimensiones, los que suelen aparecer con frecuencia hablando acerca de la violencia son emisores de otros MS (en un $62 \%$ de las ocasiones) y las instancias mediadoras (respectivamente, en un $62 \%$ y $60 \%$ de las ocasiones en las que aparece registrado su discurso sobre el MAG). Los emisores de otros MS defienden de manera inequívoca la naturaleza pacífica del MAG, posiblemente dentro de una estrategia de avalar al movimiento. Por otra parte, las posiciones

\footnotetext{
${ }^{30}$ El Gobierno acusó al PSC de "ir de la mano de Batasuna" por su coincidencia entre las organizaciones participantes en la manifestación de Barcelona.
} 
polarizadas entre las instancias mediadoras sugieren la extensión del proceso de construcción de identidad a aquellos que tienen que sopesar la legalidad de los actos de protesta y/o de las medidas represivas y preventivas.

El enfrentamiento partidista en este terreno también queda reflejado, aunque de manera más matizada, en los discursos sobre las dos dimensiones restantes analizadas. Como recoge la tabla 4, el análisis de los discursos recogidos por El País acerca de la cuestión de la representatividad del MAG ofrece algunas variaciones en el perfil de los emisores.

El sesgo de El País en la selección de discursos positivos es mayor que con relación a la dimensión anterior. Así, un $87 \%$ de los emisores subraya el carácter representativo de sus demandas. Solamente en 9 ocasiones (13\%) hemos codificado un discurso negativo en este sentido presentándolo, por ejemplo, como minoría marginal. De nuevo, el discurso del Gobierno refleja su estrategia de deslegitimación del MAG. En cuatro ocasiones este discurso es compartido por colaboradores de El País o articulistas de la sección de Opinión. No obstante, pese a que su discurso no es perfectamente uniforme, El País aparece como principal impulsor de la imagen del MAG como representativo, dando legitimidad a su causa. Las afirmaciones en este sentido de emisores del PSOE o del propio MAG son recogidas con mucha menor frecuencia.

Tabla 4.

La valoración de la representatividad del MAG en El Pais.

\begin{tabular}{lrccc}
\hline & $\begin{array}{c}\text { El MAG } \\
\text { no tiene } \\
\text { representatividad }\end{array}$ & $\begin{array}{c}\text { El MAG } \\
\text { es } \\
\text { Emisores (\% de fila) }\end{array}$ & $\begin{array}{c}\text { Núm. de } \\
\text { emisores }\end{array}$ & $\begin{array}{c}\text { \% del total } \\
\text { de emisores } \\
\text { en cada } \\
\text { categoría }\end{array}$ \\
\hline $\begin{array}{l}\text { El País (staff, editorial y } \\
\text { columnistas) }\end{array}$ & 6,1 & 93,9 & 33 & 53,2 \\
Opinión (artículos y cartas) & 28,6 & 71,4 & 7 & 46,7 \\
Gobierno/PP & 100,0 & - & 5 & 21,7 \\
PSOE & - & 100,0 & 9 & 50,0 \\
IU/LV & - & 100,0 & 2 & 66,7 \\
MAG & - & 100,0 & 5 & 22,7 \\
Otros MS & - & 100,0 & 4 & 50,0 \\
Instancias mediadoras & - & 100,0 & 1 & 20,0 \\
Otros & - & 100,0 & 4 & 41,7 \\
Total (promedio) & 12,9 & 87,1 & 70 & 41,7 \\
\hline
\end{tabular}


RIS

REVISTA INTERNACIONAL DE SOCIOLOGIA

Atendiendo al porcentaje de emisores de cada categoría que tratan esta cuestión (columna de la derecha), se observa que los partidos de izquierdas y otros MS (los aliados más directos del MAG) son los actores que, proporcionalmente, han incidido más sobre esta dimensión. En este sentido, el enfrentamiento GobiernoPSOE alrededor de esta dimensión ha sido menos intenso (o ha sido reflejado con menor intensidad en las páginas de El País).

La identidad pública del MAG se dirime también en torno a una tercera dimensión: el grado de fundamento que sostiene sus demandas (su crítica al modelo de globalización y/o sus propuestas alternativas). La tabla 5 recoge la frecuencia y la posición de los 69 emisores (el $41 \%$ del total de emisores registrados) que trataron este tema en las páginas de El País.

De acuerdo con los porcentajes en la columna de la derecha, la presencia de emisores parece estar sesgada a favor de posiciones que tienden a considerar fundamentada la crítica del MAG, y a estar de acuerdo al menos con la necesidad de reorientar el proceso de globalización. Sólo una minoría de once emisores mantiene posiciones antagónicas: las opiniones de los tres emisores del PP, cinco de los columnistas o redactores y tres autores de artículos de opinión (frente a los veinticinco y siete respectivamente, que hablan a favor del MAG en este sentido).

Dentro de las posiciones positivas, los emisores más activos en afirmar el fundamento del MAG aparecen, o bien como firmantes de artículos de opinión (en la

Tabla 5.

La valoración de la crítica del MAG al proceso de globalización en El País.

\begin{tabular}{lcccc}
\hline & \multicolumn{2}{c}{ La critica del MAG... } & & \\
\cline { 2 - 3 } Emisores (\% de fila) & $\begin{array}{c}\text {. tiene } \\
\text { fundamento }\end{array}$ & $\begin{array}{c}\text { f. carece de } \\
\text { fundamento }\end{array}$ & $\begin{array}{c}\text { Núm. de } \\
\text { emisores }\end{array}$ & $\begin{array}{c}\text { \% del total de } \\
\text { emisores en } \\
\text { cada categoría }\end{array}$ \\
\hline $\begin{array}{l}\text { El País } \\
\text { (staff, editorial y columnistas) }\end{array}$ & 83,3 & 16,7 & 30 & 48,4 \\
Opinión (artículos y cartas) & 70,0 & 30,0 & 10 & 66,7 \\
Gobierno/PP & 100,0 & 100,0 & 3 & 13,0 \\
PSOE & 100,0 & - & 4 & 22,2 \\
MAG & 100,0 & - & 13 & 59,1 \\
Otros MS & 100,0 & - & 5 & 62,5 \\
Instancias mediadoras & 100,0 & - & 1 & 20,0 \\
Otros & 84,1 & 15,9 & 69 & 20,0 \\
Total & & & & 41,1 \\
\hline
\end{tabular}


mayor parte de los casos, académicos), o como portavoces del propio MAG y de otros MS. En este debate, las pocas opiniones que recoge El País de los partidos políticos vuelven a reflejar el enfrentamiento Gobierno-PSOE, mientras que IU, que por lo general recibe escasa cobertura en El País, no figura en esta ocasión como emisor.

En resumen, como refleja el perfil de los emisores, la naturaleza violenta del MAG es la dimensión que más claramente refleja la politización del proceso de construcción de la imagen del MAG. El creciente apoyo de los partidos tradicionales de izquierdas, como se reflejó en el Foro de Porto Alegre, se ha visto favorecido en España por la situación del PSOE en la oposición en un momento de creciente movilización social contra las políticas conservadoras (reforma de la ley universitaria, del desempleo, etc.). Dicha politización implica la alianza coyuntural de actores institucionalizados (PSOE) y El País, frente a la imagen que promueve el Gobierno del MAG como un movimiento violento, que justifica las medidas represivas preventivas (las medidas de seguridad).

En este contexto, en el transcurso del semestre el discurso del Gobierno se presenta de manera creciente como una estrategia de criminalización del MAG. La tabla 6 ofrece información sobre el número de emisores que incorporan en su discurso el tema de la criminalización. Un 38\% de los emisores, en casi el $50 \%$ de las NPE en las que se construye la identidad del MAG, habla de la existencia de dicha estrategia. En comparación con los emisores que tratan las tres dimensiones de la identidad del MAG analizadas anteriormente, aparece con mayor frecuencia en el tiempo, pasando de ser menos frecuente que cualquiera de ellas en marzo, a ser, al final del proceso, la cuestión más tratada (i.e., parece que las acusaciones de "violento" son crecientemente interpretadas como parte de una estrategia de criminalización por parte del Gobierno).

Como en las tablas anteriores, El País vuelve a aparecer como el principal impulsor de un discurso que señala la existencia de un proceso de criminalización del MAG, siendo en relación con las dimensiones anteriores un aspecto tratado de manera especialmente frecuente por los autores de artículos de opinión (un $66 \%$ de los mismos hablan de criminalización). El PSOE, aunque también participa de este discurso, es menos activo que en su negación del carácter violento del movimiento o en la afirmación de su legitimidad. Podría decirse que el enfrentamiento con el PP no se ha materializado en estos términos con tanta frecuencia como en relación con otras dimensiones del discurso sobre la naturaleza del MAG. De acuerdo con estos resultados, y teniendo en cuenta su inferior acceso, los impulsores de este discurso han sido fundamentalmente el resto de los partidos de izquierdas y emisores vinculados a otros MS, mucho más activos en los procesos de movilización que el partido socialista. 
RIS

REVISTA INTERNACIONAL DE SOCIOLOCIA

№ 33, Septiembre-Diciembre, 2002

MANUEL JIMÉNEZ y JAVIER ALCALDE

Tabla 6.

Los promotores del discurso de denuncia de la criminalización del MAG.

\begin{tabular}{lcc}
\hline Emisores (\% de fila) & $\begin{array}{c}\text { Número de Emisores } \\
\text { que hablan de la } \\
\text { criminalización }\end{array}$ & $\begin{array}{c}\% \text { del total } \\
\text { de emisores en cada } \\
\text { categoria }\end{array}$ \\
\hline El País (staff, colabora y editorialistas) & 23 & 37,1 \\
Público en El País (opinión, cartas) & 10 & 66,7 \\
PSOE & 5 & 27,8 \\
IU/LV & 2 & 66,7 \\
MAG & 8 & 36,4 \\
Otros MS & 5 & 62,5 \\
Instancias mediadoras & 2 & 40,0 \\
Otros & 8 & 66,7 \\
Total & 63 & 37,5 \\
\hline
\end{tabular}

\section{CONCLUSIONES}

El semestre de la presidencia española de la UE ofrece una buena oportunidad para examinar la presencia del MAG en los medios de comunicación y, en concreto, analizar el proceso de construcción de su identidad pública. En las siguientes conclusiones sintetizamos qué nos dicen nuestros resultados sobre este fenómeno. A este respecto, es oportuno señalar que la naturaleza de dichos resultados se ajusta a las predicciones establecidas por la literatura sobre cómo operan los medios y la elaboración de noticias por lo que, consecuentemente, presentan una serie de sesgos que deben ser tenidos en cuenta.

De acuerdo con nuestras expectativas, la presencia mediática del MAG está vinculada a la cuestión de la violencia. En este sentido, cabe señalar al menos tres resultados de naturaleza empírica. En primer lugar, hay que destacar el amplio volumen de noticias sobre la seguridad durante las cumbres y la altísima frecuencia de menciones al MAG. La respuesta de las autoridades a la extensión de la contestación al modelo de globalización, posiblemente estimulada por el devenir político tras el 11-S, se ha centrado en la seguridad. No obstante, en segundo lugar, esta circunstancia no ha impedido la consolidación en la agenda política (y mediática) del debate sobre la globalización, como indica el alto porcentaje de noticias que tratan la cuestión. Desde esta perspectiva, el éxito del MAG es innegable y refleja cómo, en distinta medida, todo el espectro de la izquierda tradicional ha incorporado su discurso, como indica la participación de organizaciones políticas y sindicales tradicionales en el Foro Social Barcelona. Estas dos respuestas desde 
los actores políticos institucionalizados, la "negativa" basada en la seguridad y la "positiva" que incorpora (parte de) su discurso, explican un tercer resultado destacable: la intensidad del proceso de construcción de la identidad pública del MAG en los medios. El carácter conflictivo de este proceso queda reflejado, con los sesgos que introduce el criterio de selección del periódico, en la configuración del mapa de actores que se pronuncian a favor y en contra del MAG. Desde una perspectiva temporal, también se puede hablar de un momento de inflexión, después del éxito de convocatoria en los actos de protesta de Barcelona, a partir del que decrece la intensidad de este proceso. Además, en términos relativos, el debate sobre su naturaleza violenta va perdiendo relevancia a favor del discurso que denuncia la existencia de una estrategia de criminalización o la discusión sobre el fundamento de sus demandas. Estos resultados indican, en la interpretación propuesta, un progresivo distanciamiento de la imagen violenta del MAG y, por tanto, un avance en la configuración positiva de su identidad pública. En general, desde una perspectiva teórica, estos resultados respaldan las conclusiones de trabajos previos (Sampedro, 1997) que vinculan la visibilidad mediática del los MS, por un lado, a su capacidad de interpelación e interlocución con actores políticos institucionalizados y, por otro lado, a su encaje en la línea editorial dentro de los apoyos mediático-partidarios.

Como hemos señalado anteriormente, estas conclusiones se basan en una aproximación parcial al proceso de configuración de la identidad del MAG. A este respecto, la lectura de las conclusiones anteriores debe incorporar, al menos, dos consideraciones. En primer lugar, el semestre analizado se sitúa en un momento temporal y geográficamente concreto. El apoyo, más o menos implícito, del PSOE al movimiento, por ejemplo, aunque puede insertarse en la tendencia general de acercamiento de la socialdemocracia al MAG (como se puso de manifiesto en el Foro Social de Porto Alegre), se ha visto favorecido por el contexto político de enfrentamiento con el Gobierno en un momento de amplia movilización social, motivada por las reformas en materia de desempleo y educación. Igualmente, resulta razonable interpretar la ausencia de violencia antiglobalización durante el semestre, esencialmente como consecuencia del predominio entre los MS en España de una cultura de protesta pacífica (Jiménez, 2002). Esta explicación plantea otro interrogante a nuestra afirmación sobre la consolidación de la imagen pacífica del MAG fuera de nuestras fronteras o en el futuro. En segundo lugar, y quizás más importante, nuestras conclusiones están sesgadas por la visión de la realidad emitida desde El País. En este sentido, los resultados no reflejan la construcción de la identidad pública del MAG en el conjunto de la opinión pública española. Es razonable pensar que la incorporación de otros medios como fuentes a este tipo de análisis, modificaría algunos de los resultados, como los relativos al nivel de acceso de los distintos emisores, y matizaría nuestra interpretación sobre la evolución positiva de la imagen del MAG y sobre su alto nivel de acceso a los medios. No obstante, nuestros datos apuntan a que la mayoria de las conclusiones, como las 
R I S

REYISTA INTERNACIONAL DE SOCIOLOCIA

relativas a la importancia del tema de la seguridad, y las continuas referencias al MAG y a su identidad, seguirían siendo válidas con la inclusión en el análisis de las noticias publicadas en otros medios.

\section{REFERENCIAS BIBLIOGRÁFICAS}

CHILTON, P. y C. SCHÄFFNER (2000), "Discurso y política", en van Dijk, T. A. (comp.), El Discurso como interacción social, Barcelona, Gedisa Editorial, pp. 297-329.

ERICSON R. y A. DOYLE (1999), "Globalization and the policing of protest: the case of APEC 1997”, British Journal of Sociology, $\mathrm{n}^{\circ}$ 50, (4), pp. 489-608.

FERNÁNDEZ DURÁN, R.M., M. ETXEZARRETA y M. SÁEZ (2001), Globalización Căpitalista. Luchas y Resistencias, Barcelona, Virus Editorial.

GAMSON, W. (1990), The Strategy of Social Protests (2 edición), Belmont, CA., Wadsworth Publishing Company.

GAMSON, W. y G. WOLSFIELD (1993), "Movements and Media as Interacting Sytems", ANNALS, AAPSS, $\mathrm{n}^{\circ}$ 528, pp. 114-125.

GERHARDS J. y D. RUCHT. (1992), "Mesomobilization: Organizing and Framing in two Protest Campaigns in West Germany", American Journal of Sociology, no 98 (3), pp. 555-595.

GRANT, J. y W. MALONEY (1997), The Protest Business? Mobilizing Campaign Groups, Manchester, Manchester University Press.

HALLORAN, J.D., P. ELLIOT y G. MURDOCK (1970), Demonstrations and Communication: A case Study, Middlesex, England, Penguin Books Inc.

HOCKE, P. (1998), "Determining the Selection Bias in Local and National Newspaper Reports on Protests Events", en Rucht, D., R. Koopmans y F. Neidhardt (eds.), Acts of Dissent, Lanham, Md, Rowman \& Littlefield.

HUG, S. y D. WISLER (1998), "Correcting for the Selection Bias in Social Movement Research", Mobilization, 3(2), pp. 141-161.

JIMÉNEZ, M. (2002), Protesta Social y Políticas Públicas. Un Estudio de la Relación entre el Movimiento Ecologista y la Politica Ambiental en España. Colección Tesis Doctorales, $\mathrm{n}^{\circ} 34$, Madrid, Instituto Juan March.

JOHNSTON, H., E. LARAÑA y J.R. GUSFIELD (1994), "Identities, Grievances, and New social Movements" en Laraña, E., H. Johnston y J. R. Gusfield (eds.), New Social Movements: from Ideology to Identity, Philadelphia, Temple University Press.

KIELBOWICZ, R. B. y C. SCHERER (1986), "The Role of the Press in the Dynamics of Social Movements", Research in social Movements, Conflict and Change, $\mathrm{n}^{\circ}$ 9, pp. 71-96. 
MINKOFF, D.C. (1997), "Producing Social Capital. National Social Movements and Civil Society", American Behavioral Scientist, $\mathrm{n}^{\circ} 40$ (5), pp. 606-619.

MOLTOCH, H. (1977), "Media and Movements", en Zald, M.N. y J. McCarthy (Eds.), The Dynamics of Social Movements. Resource Mobilization, Social Control, and Tactics, Cambridge, Massachusetts, Winthrop Publishers Inc.

PAGE, B. I. (1996), Who Deliberates?: Mass Media in Modern Democracy, Chicago, Ill, University of Chicago Press.

PASTOR, J. (2002), ¿Qué son los movimientos antiglobalización?, Barcelona, RBA Libros.

RUCHT, D., R. KOOPMANS y F. NEIDHARDT (eds.) (1998), Acts of Dissent. New Developments in the Study of Protest, Berlin, Edition Sigma.

SAMPEDRO, V. (1997), Debates sin Mordaza: Desobediencia civil y servicio militar, 1970-1996, Madrid, Boletín Oficial del Estado; Centro de Estudios Constitucionales.

SMITH, J. (2001), "Globalizing Resistance: The Battle of Seattle and the Future of Social Movements", Mobilization, nº 6 (1), pp.1-19.

SNYDER, D. y W. R. KELLY (1977), "Conflict intensity, media sensitivity and the validity of newspaper data", American Sociological Review, n 40, pp. 259-278.

TARROW, S. (1998), Power in Movement (2a edición), Cambridge/Nueva York, Cambridge University Press.

VAN DIJK, T. A. (1996), La Noticia como Discurso. Compresión, estructura y producción de la información, Barcelona, Ediciones Paidós.

VAN ZOONEN, E.A. (1992), "The Women's Movement and the Media: Constructing a Public Identity", European Journal of Communication, n 7, pp. 453-476.

WALGRAVE S. y J. MANSSENS (2000), "The Making of the White March: The Mass Media as a Mobilizing Alternative to Movement Organizations", Mobilization, $n^{\circ} 5$ (2), pp. 217-239. 